\title{
Ultra-High Gradient Dielectric Wakefield Accelerator Experiments
}

M. C. Thompson, H. Badakov, J. B. Rosenzweig, G.

Travish, M. Hogan, R. Ischebeck, N. Kirby, R. Siemann, D. Walz, P. Muggli, A. Scott, R. Yoder

\section{August 8, 2006}

Advanced Accelerator Concepts Workshop 2006 Lake Geneva, WI, United States July 10, 2006 through July 15, 2006 
This document was prepared as an account of work sponsored by an agency of the United States Government. Neither the United States Government nor the University of California nor any of their employees, makes any warranty, express or implied, or assumes any legal liability or responsibility for the accuracy, completeness, or usefulness of any information, apparatus, product, or process disclosed, or represents that its use would not infringe privately owned rights. Reference herein to any specific commercial product, process, or service by trade name, trademark, manufacturer, or otherwise, does not necessarily constitute or imply its endorsement, recommendation, or favoring by the United States Government or the University of California. The views and opinions of authors expressed herein do not necessarily state or reflect those of the United States Government or the University of California, and shall not be used for advertising or product endorsement purposes. 


\title{
Ultra-High Gradient Dielectric Wakefield Accelerator Experiments
}

\author{
M.C. Thompson*, H. Badakov ${ }^{\dagger}$, J.B. Rosenzweig ${ }^{\dagger}$, G. Travish ${ }^{\dagger}$, M. \\ Hogan $^{* *}$, R. Ischebeck ${ }^{* *}$, N. Kirby**, R. Siemann**, D. Walz ${ }^{* *}$, P. Muggli ${ }^{*}$, \\ A. Scott ${ }^{\S}$ and R. Yoder ${ }^{\mathbb{I}}$ \\ * Lawrence Livermore National Laboratory, Livermore, California, 90095 \\ ${ }^{\dagger}$ UCLA Department of Physics and Astronomy, Los Angeles, California, 90095 \\ ** Stanford Linear Accelerator Center, Stanford, California, 94309 \\ ${ }^{\ddagger}$ University of Southern California Los Angeles, California, 90089 \\ ${ }^{\S}$ UCSB Department of Physics, Santa Barbara, California, 93106 \\ IManhattan College, Riverdale, New York, 10471
}

\begin{abstract}
Ultra-high gradient dielectric wakefield accelerators are a potential option for a linear collider afterburner since they are immune to the ion collapse and electron/positron asymmetry problems implicit in a plasma based afterburner. The first phase of an experiment to study the performance of dielectric Cerenkov wakefield accelerating structures at extremely high gradients in the $\mathrm{GV} / \mathrm{m}$ range has been completed. The experiment took advantage of the unique SLAC FFTB electron beam and its ultra-short pulse lengths and high currents (e.g., $\sigma_{z}=20 \mu \mathrm{m}$ at $\mathrm{Q}=3 \mathrm{nC}$ ). The FFTB electron beam was successfully focused down and sent through short lengths of fused silica capillary tubing (ID $=200 \mu \mathrm{m} / \mathrm{OD}=325 \mu \mathrm{m}$ ). The pulse length of the electron beam was varied to produce a range of electric fields between 2 and $20 \mathrm{GV} / \mathrm{m}$ at the inner surface of the dielectric tubes. We observed a sharp increase in optical emissions from the capillaries in the middle part of this surface field range which we believe indicates the transition between sustainable field levels and breakdown. If this initial interpretation is correct, the surfaced fields that were sustained equate to on axis accelerating field of several $\mathrm{GV} / \mathrm{m}$. In future experiments being developed for the SLAC SABER and BNL ATF we plan to use the coherent Cerenkov radiation emitted from the capillary tube as a field strength diagnostic and demonstrate $\mathrm{GV} / \mathrm{m}$ range particle energy gain.
\end{abstract}

Keywords: acceleration, wakefield accelerators, dielectric devices, Cherenkov radiation PACS: $41.75 . \mathrm{Lx}, 41.60 . \mathrm{Bq}, 41.75 . \mathrm{Ht}, 77.22 . \mathrm{Jp}$

\section{INTRODUCTION}

The accelerating field achievable in conventional radio frequency accelerators is ultimately limited by the breakdown of the metallic accelerating structure. It is well understood that the field sustainable in a cavity increases with the frequency of the cavity $[1,2]$. Simply following this scaling by using ever higher frequency cavities is difficult due to the "THz gap"; the current lack of practical high power source between X-band microwaves $(\sim 11 \mathrm{GHz})$ and infrared lasers $(\sim 28 \mathrm{THz})$. Various studies have indicated that $\mathrm{GV} / \mathrm{m}$ accelerating fields should be possible in dielectric laser accelerators [3] as long as the driving laser pulses are very short [4]. The difficulty with laser accelerators, however, is that they operate at very short wavelengths (e.g. $10.6 \mu \mathrm{m}$ at $28 \mathrm{THz}$ ), which greatly complicates beam injection and phasing.

Dielectric accelerators can also be powered directly by high energy charged particle 


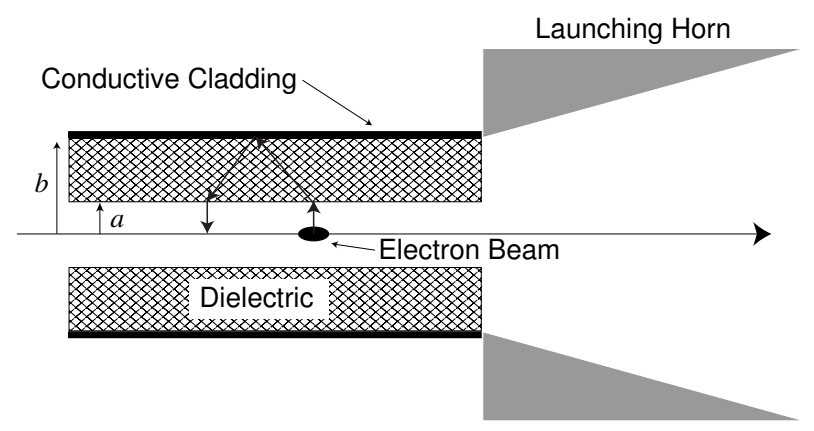

FIGURE 1. Schematic of the dielectric wake experiment.

beams via wakefield excitation [5, 6], eliminating the need to generate high power electromagnetic waves, and can operate at essentially any wavelength since the accelerating wakefield wavelength is determine by the dielectric geometry. An electron-beam-driven dielectric-wakefield-accelerator is essentially a uniform dielectric tube coated on the outside with a conductor, see Fig. 1. When an intense electron beam passes through the center of the tube its electric field bends at the Cerenkov angle within the dielectric, reflects off the outside conducting layer, and returns to the axis where it can be used to accelerate other particles. In the ideal limit, where the electron beam length $\left(\sigma_{z}\right)$ is much smaller than the the inner dielectric radius $(a)$, the on axis accelerating field $\left(E_{z, a c c e l}\right)$ is given by $E_{z, \text { accel }} \approx 2 Q / a^{2}$, where $Q$ is the beam charge. Maximizing the accelerating wakefield therefore requires maximizing $Q$ while minimizing $\sigma_{z}$ and $a$. It should also be noted that $a$ must be chosen several times larger than the beam radius $\left(\sigma_{r}\right)$ to prevent damage to the dielectric from the beam halo.

Dielectric wakefield accelerators have been studied in depth over the last several years, but their maximum fields have been limited to 10 's of $\mathrm{MeV} / \mathrm{m}$ by the lack of ultra-short drive beams. The recently achieved $20 \mu \mathrm{m}$ pulse-length beams obtained at the Stanford Linear Accelerator Center (SLAC) Final Focus Test Beam (FFTB) facility are sufficient to generate longitudinal fields in excess of $1 \mathrm{GV} / \mathrm{m}$. We have used the FFTB beam to begin probing the $\mathrm{GV} / \mathrm{m}$ regime of dielectric accelerators.

\section{BREAKDOWN THRESHOLD OBSERVATIONS}

The primary goal of the first phase of the experiment was to assess dielectric material survivability. The parameters for our experiment, which is based upon the unprecedented combination of high charge, short pulse duration, and small spot size available in the SLAC FFTB beam, are listed in Table 1. The peak electric field values in Table 1 are derived from OOPIC simulations [7], see Fig. 2. The ability to push surface fields up to $22 \mathrm{GV} / \mathrm{m}$ virtually guaranteed that we would be able to break down the dielectric.

Since the experiment uses dielectric tubes with 100 and $200 \mu \mathrm{m}$ IDs, we limited the tube length to about a $\mathrm{cm}$ in order to mitigate alignment issues. The energy gained or lost by beam particles was therefore only on the order of $10 \mathrm{MeV}$, while the full energy spread of the highly compressed FFTB beam is several GeV. Consequently, energy 
TABLE 1. Breakdown Experiment Parameters

\begin{tabular}{lclc}
\hline Beam Charge $(Q)$ & $3 \mathrm{nC}$ & Outer Dielectric Radius $(b)$ & $162 \mu \mathrm{m}$ \\
Beam Energy & $30 \mathrm{GeV}$ & Dielectric Relative Permittivity $(\varepsilon)$ & $\sim 3$ \\
Beam Radius $\left(\sigma_{r}\right)$ & $10 \mu \mathrm{m}$ & Peak Decelerating Field & $8 \mathrm{GV} / \mathrm{m}$ \\
Beam Length $\left(\sigma_{z}\right)$ & $100-20 \mu \mathrm{m}$ & Peak Accelerating Field & $12 \mathrm{GV} / \mathrm{m}$ \\
Inner Dielectric Radius $(a)$ & 50 and $100 \mu \mathrm{m}$ & Peak Field at Dielectric & $22 \mathrm{GV} / \mathrm{m}$ \\
\hline
\end{tabular}

changes were not resolvable in this experiment. The primary signature of high field wakes in this first phase of the experiment was the detection of dielectric breakdown.

This dielectric wakefield experiment is unique in that it produced fields comparable to those in earlier laser breakdown studies. We are particularly interested in differentiating material breakdown in wake-excited, relatively long wavelength $(>50 \mu \mathrm{m}$ photon) systems, as compared with optically (laser) excited systems. Quantum absorption of photons to produce free electrons in the material is known to be an initiator of avalanche ionization [4]. This mechanism should be mitigated in the wake experiment, since the individual photon energies are lower than in the laser case, perhaps allowing higher fields to be tolerated. In the case of optically excited fields, the breakdown limit for a 70 fs pulse is less than $2 \mathrm{GV} / \mathrm{m}$ in fused silica [4]. As discussed below, it seems that surface fields higher than $2 \mathrm{GV} / \mathrm{m}$ where sustained in our dielectric wake experiment at similar pulse lengths.

The dielectric tubes for this experiment were modified from off-the-shelf synthetic fused silica capillary tubing ( $\varepsilon \cong 3$ ), which we procured in 100 and $200 \mu \mathrm{m}$ ID sizes. Samples were prepared from this bulk product by cutting pieces to length, removing the outer polyimide jacket, polishing the cut ends, and coating the outside of the tube with vapor deposited aluminum. Groups of ten samples were packaged together for the experiment in modular mounting blocks. The $1 \mathrm{~cm}$ wide, by $5 \mathrm{~cm}$ long, by $0.5 \mathrm{~cm}$ high

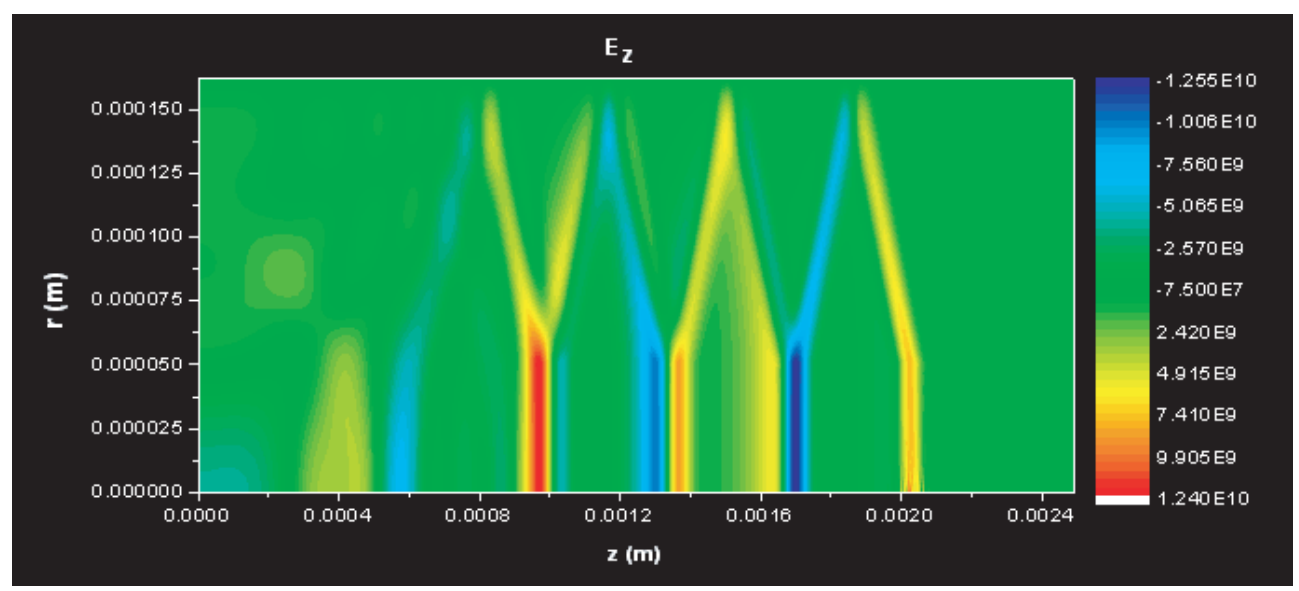

FIGURE 2. OOPIC simulation results for parameters $Q=3 \mathrm{nC}$, beam energy $30 \mathrm{GeV}, \sigma_{r}=10 \mu \mathrm{m}, \sigma_{z}$ $=20 \mu \mathrm{m}, a=50 \mu \mathrm{m}, b=162 \mu \mathrm{m}$, and $\varepsilon=3$. This contour plot shows the z component of the electric field $\left(E_{z}\right)$ throughout the simulation region. The electron pulse is moving the right and its centroid coincides with the sharp peak of $E_{z}$ near $z=2 \mathrm{~mm}$. Note how the wake oscillations retain the sharply peaked field distribution. 

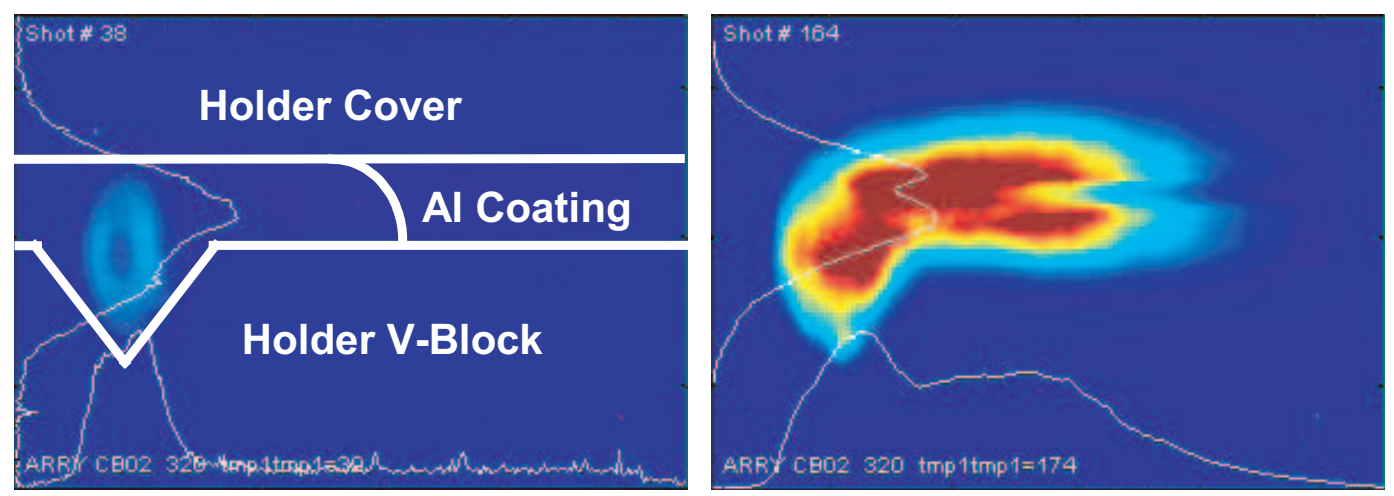

FIGURE 3. Visible light images (displayed with the same scaled-to-intensity color map) of a $200 \mu \mathrm{m}$ ID fiber tip as the electron beam pulse current is ramped up. In the image at left, the electron beam pulse length is long $\left(\sigma_{z} \sim 100 \mu \mathrm{m}\right)$ and the fiber end glows faintly with incoherent optical wavelength emissions. The various boundaries of the fiber holder, which is viewed at an angle of $45^{\circ}$ from the beam axis, are also illustrated in the left hand frame along with the area in which the fiber's aluminum coating blocks optical emissions. In the right hand frame the electron beam is near its minimum pulse length $\left(\sigma_{z}\right.$ $\sim 20 \mu \mathrm{m}$ ) and a breakdown event has occurred producing a large flash of visible light.

mounting block also has an optical transition radiation (OTR) screen for locating the beam orbit. Our methodology for aligning the experiment was to move the dielectric tubes onto the established beam orbit rather than trying to steer the beam through the tubes. In practice, this alignment technique seemed to work very well, although we could not rule out beam halo scrapping in the $1 \mathrm{~cm}$ long dielectric tubes. We intended to check for scrapping by monitoring the x-rays such scrapping would produce, but discovered that our X-ray detector was too insensitive. Alignment procedures will be further refined and use better $x$-ray diagnostics in future runs of the dielectric wake experiment. Once beam propagation through the dielectric tubes was established, we began to vary the wakefield magnitude by adjusting the beam pulse length and search for an optical signal of breakdown on a side view telescopic camera. This experiment was repeated for samples with different tube IDs. Individual tube samples were systematically exposed to different field levels for varying periods of time for a later study of differences in material damage.

While analysis of the collected data is still preliminary, the observations seem to indicate a clear breakdown threshold. Most of the observations were of $200 \mu \mathrm{m}$ ID fibers and the general impression is that the visible light output of the fibers jumped up sharply in the middle of the beam pulse length range, Fig. 3. This signal was clearly visible on both the side view telescopic camera and a wide-angle top-view general observation camera. The initiation of breakdown discharges are most likely responsible for the optical radiation increase. The visible wavelength light recorded at long pulse length / low fields is probably due to a combination of incoherent cerenkov radiation, incoherent transition radiation, and scintillation. Once a breakdown occurs, however, the light from these mechanisms is small compared to the visible emissions from the plasma formed during the breakdown event. It is clear from the observations that breakdown events start to occur after the beam has been compressed below the midpoint of its range. For an ultra-relativistic beam $(\gamma \gg 1)$ it is fairly straightforward to calculate the 


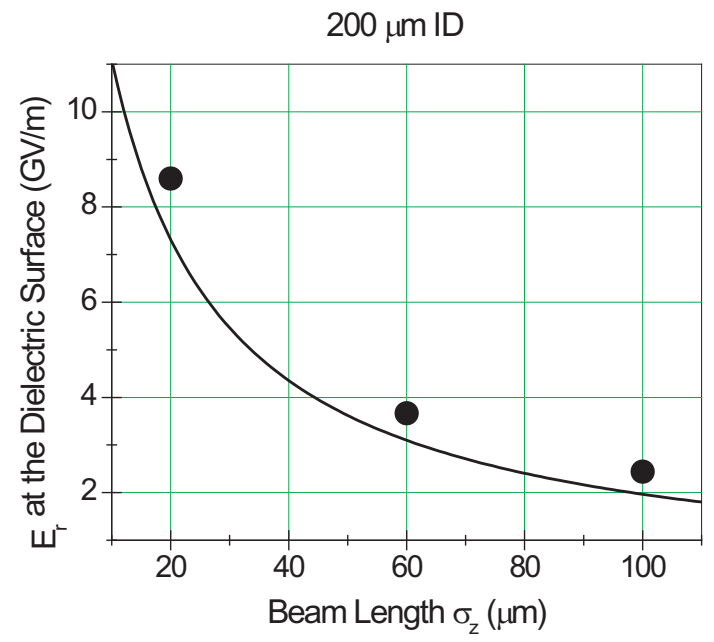

FIGURE 4. Radial electric field at the inner surface of a $200 \mu \mathrm{m}$ ID fused silica capillary tube as a function of electron beam pulse length. The line is the prediction of Eq. 1 and the dots are the results of OOPIC simulations. Note that the slight offset arises because the OOPIC results give the peak field while Eq. 1 give the average field over the effective length of the beam. It is not yet clear which method of evaluating the surface fields produces a better figure of merit.

radial field on the surface of the dielectric $E_{r, \text { surface }}$ to first approximation through simple application of Gauss' law. Such an analysis yields the expression

$$
E_{r, \text { surface }}=\frac{1}{\varepsilon_{0}} \frac{Q}{(2 \pi)^{3 / 2} a \sigma_{z}+\frac{\sqrt{\varepsilon-1}}{\varepsilon} \pi a^{2}}\left[\frac{\mathrm{V}}{\mathrm{m}}\right],
$$

where all quantities are in SI units, $\varepsilon_{0}=8.854 \times 10^{-12} \mathrm{~F} \mathrm{~m}^{-1}$ is the permittivity of free space, and $\varepsilon$ is the dielectric constant. This result compares well to those of more sophisticated analytical theory [8,9]. Using Eq. 1 to plot the surface fields at the dielectric verses the beam pulse length, Fig. 4, we find that at the midpoint of beam compression $\left(\sigma_{z} \sim 60 \mu \mathrm{m}\right)$ where breakdown is observed the surface fields at the dielectric are in the range $3-4 \mathrm{GV} / \mathrm{m}$. OOPIC simulations indicated that this range of surface fields corresponds to $1.5-2 \mathrm{GV} / \mathrm{m}$ on-axis accelerating fields in the wake.

Further analysis of the data will allow us to make a more accurate determination of the fused silica capillary tube breakdown threshold. In addition to a more careful analysis of the visible light observations and associated beam parameters, we also intend to examine the dielectric tubes with various diagnostics to detect structural damage from the breakdown events. We hope to be able to correlate the number of breakdown events a particular tube experienced to the degree of pitting or other forms of damage that are evident in the postmortem analysis. Unfortunately, the second phase of the experiment, which planned to measure the coherent Cerenkov radiation emitted by the fiber as an independent indicator of field strength $[10,11]$, is on indefinite hold after the decommissioning of the FFTB in spring 2006. Further work must wait for the commissioning of the proposed SABER facility at SLAC [12]. 


\section{TOWARDS A DIELECTRIC AFTERBURNER}

The concept of building a energy doubling "afterburner" for an $\mathrm{e}^{+} \mathrm{e}^{-}$linear collide [13] has been a central goal in particle beam driven plasma wakefield accelerator (PWFA) research for several years. The primary attraction of the afterburner concept is the huge accelerating fields made possible by PWFAs. A recent experiment demonstrated 27 $\mathrm{GV} / \mathrm{m}$ acceleration over $10 \mathrm{~cm}$ with a electron beam driven PWFA [14]. Unfortunately, the operation of a PWFA is not symmetric under charge reversal and much less progress has been made in the more complex case of the positron PWFA. Also, it has recently been shown that motion of the plasma ions, which were previously assumed to be immobile, endangers the feasibility of a PWFA afterburner for International Linear Collider (ILC) beam parameters [15]. While the difficulties of a PWFA afterburner may be overcome, the problems are serious enough to merit the examinations of alternative afterburner concepts.

We propose that a afterburner could be constructed with dielectric wakefield accelerators and that experiments at the SLAC SABER facility can test the feasibility of this concept. While a dielectric afterburner could not achieve accelerating field as high as a PWFA, our preliminary breakdown study results indicate the accelerating fields of at least $1 \mathrm{GV} / \mathrm{m}$ are possible. $1 \mathrm{GV} / \mathrm{m}$ is well over an order of magnitude higher than the ILC design gradient. Most importantly, however, a dielectric afterburner would be symmetric for electrons and positrons and would be immune to the problems associated with ion collapse.

The initial SABER experiments designed to examine the dielectric afterburner concept will be of the energy gain / loss variety. Our general concept is to send weakly compressed electron and positron beams of identical parameters through a long $(\sim 10$ $\mathrm{cm}$ ) dielectric tube designed to give a gradient $>1 \mathrm{GV} / \mathrm{m}$ and examine both beams for head to tail energy modulation. An example set of experimental parameters is listed in Table 2. OOPIC simulations, Fig. 5, show that the long electron beam will interact with the entire first period of its wake. Such an experiment would demonstrate high-gradient polarity-symmetric acceleration in a dielectric wakefield accelerator and is well within the planned capabilities of SABER and dielectric structures we are developing.

TABLE 2. SABER Polarity Symmetric Energy Gain Experiment Parameters

\begin{tabular}{lclc}
\hline Beam Charge* $^{*} 5 \mathrm{nC}$ & Inner Dielectric Radius $(a)$ & $100 \mu \mathrm{m}$ \\
Beam Energy & $30 \mathrm{GeV}$ & Outer Dielectric Radius $(b)$ & $150 \mu \mathrm{m}$ \\
Energy Spread (FWHM) & $<0.25 \%$ & Dielectric Relative Permittivity $(\varepsilon)$ & $\sim 3$ \\
& $(.075 \mathrm{GeV})$ & Dielectric Length & $10 \mathrm{~cm}$ \\
Beam Radius $\left(\sigma_{r}\right)$ & $10 \mu \mathrm{m}$ & Peak Accelerating Field & $1.1 \mathrm{GV} / \mathrm{m}$ \\
Beam Length $\left(\sigma_{z}\right)$ & $150 \mu \mathrm{m}$ & Maximum Energy Gain & $0.11 \mathrm{GeV}$ \\
\hline
\end{tabular}

* All parameters are for both $\mathrm{e}^{-}$and $\mathrm{e}^{+}$beams 


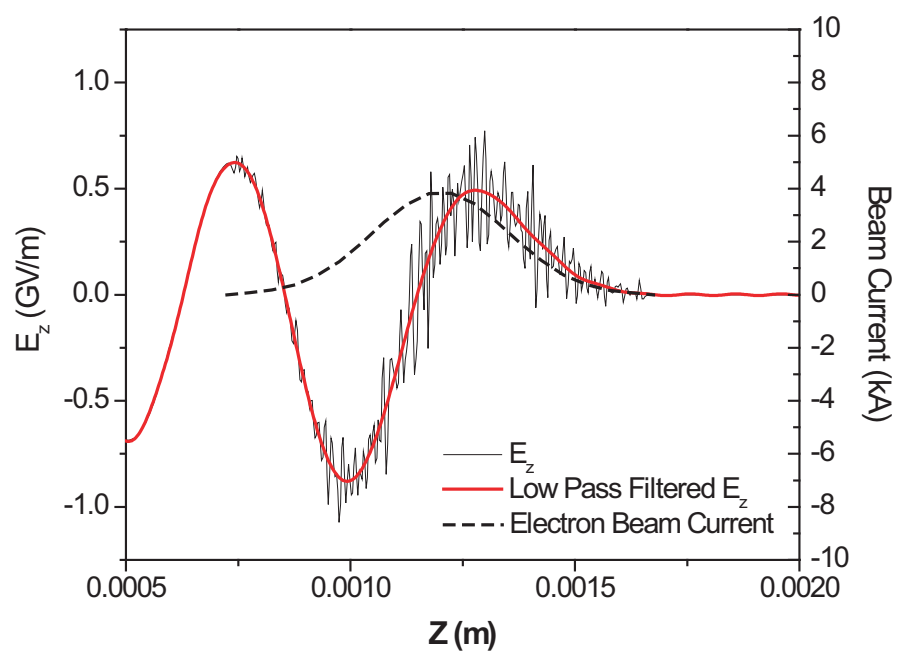

FIGURE 5. OOPIC simulation results for the parameters listed in Table 2. The plot shows a line out of the $z$ component of the electric field $E_{z}$ at a radius of $10 \mu \mathrm{m}$ along with the beam current. Note that, unlike the case shown in Fig. 2, in this scenario $\sigma_{z}>a$ which and leads to broad sinusoidal wakefields which overlap with the driving beam.

\section{ACKNOWLEDGMENTS}

This work was performed under the auspices of the US Department of Energy under Contract No. DE-FG03-92ER40693 and W-7405-ENG-48.

\section{REFERENCES}

1. W. D. Kilpatrick, Review of Scientific Instruments 28, 824 (1957).

2. G. A. Loew, and J. W. Wang, "RF Breakdown and Feild Emission", Tech. Rep. SLAC-PUB-4845, SLAC (1989).

3. J. Rosenzweig, A. Murokh, and C. Pellegrini, Phys. Rev. Lett. 74, 2467-2470 (1995).

4. D. Du, X. Liu, G. Korn, J. Squier, and G. Mourou, Appl. Phys. Lett. 64, 3071 (1994).

5. W. Gai, et al., Phys. Rev. Lett. 61, 2756 (1988).

6. J. G. Power, et al., Phys. Rev. ST Accel. Beams 3, 101302 (2000).

7. D. L. Bruhwiler, et al., Phys. Rev. ST Accel. Beams 4, 101302 (2001).

8. R. Siemann, and A. Chao, "Wakefields in a Dielectric Tube with Frequency Dependent Dielectric Constant", Tech. Rep. ARDB-368, SLAC (2004).

9. R. H. Siemann, "Decelerating Gradient for FFTB Dielectric Tube Experiment", Tech. Rep. ARDB371, SLAC (2004).

10. J. B. Rosenzweig, et al., "An Ultra-High Gradient Cerenkov Wakefield Acceleration Experiment at SLAC FFTB," in Advanced Accelerator Concepts: Eleventh Workshop, 2004, vol. 737 of AIP Conf. Proc., p. 811.

11. M. C. Thompson, et al., "The UCLA/SLAC Ultr-High Gradient Cerenkov Wakefield Accelerator Experiment," in Proc. PAC 2005, IEEE, 2005, p. 3067.

12. M. Hogan, et al., these proceedings.

13. S. Lee, et al., Phys. Rev. Special Topics - Accel. Beams 5, 011001 (2002).

14. M. J. Hogan, et al., Phys. Rev. Lett. 95, 054802 (2005).

15. J. B. Rosenzweig, A. M. Cook, A. Scott, M. C. Thompson, and R. B. Yoder, Phys. Rev. Lett. 95, 195002 (2005). 